\title{
Outcome results in children with IgA nephropathy: a single center experience
}

This article was published in the following Dove Press journal: International Journal of Nephrology and Renovascular Disease 19 January 2012

Number of times this article has been viewed

\author{
Ipek Kaplan Bulut' \\ Sevgi Mir ${ }^{\prime}$ \\ Betul Sozeri' \\ Mustafa Orhan Bulut ${ }^{2}$ \\ Sait Sen ${ }^{3}$ \\ Nida Dincel' \\ 'Ege University Faculty of Medicine \\ Department of Pediatric Nephrology, \\ ${ }^{2}$ Ege University Faculty of Medicine \\ Department of Pediatrics, Izmir, \\ ${ }^{3}$ Ege University Faculty of Medicine \\ Department of Pathology, \\ Izmir, Turkey
}

Background: Immunoglobulin A nephropathy (IgAN) is the most common primary glomerulonephritis. Patients manifest variable clinical symptoms (eg, microhematuria) with preserved or progressive deterioration of renal function resulting in end-stage renal disease. The aim of this study was to evaluate patients from a single center to describe the clinical features, treatments, and follow-up results of those with the disease.

Methods: This is a retrospective data study of all children with IgAN. Patients who had a histopathologically proven diagnosis of IgAN and were followed up for at least 5 years were included in the study. Renal biopsy, graded as Hass classification, was performed on all patients. A total of 39 patients were included in the study.

Results: The mean follow-up time ( \pm standard deviation) was $10.4 \pm 3.51$ (range 5-16) years. Twenty-nine patients $(74.4 \%)$ were male and ten $(25.6 \%)$ were female. Nineteen $(48.7 \%)$ patients presented with recurrent macroscopic hematuria, ten $(25.6 \%)$ with microscopic hematuria \pm proteinuria, six (15.4\%) with nephritic syndrome, and four $(10.3 \%)$ with nephrotic syndrome. All patients underwent a renal biopsy, which was graded according to the Hass classification. At the end of follow-up time, 18 (46.1\%) patients were normal, 15 (38.5\%) had minor urinary abnormalities, three (7.7\%) had active renal disease, and three (7.7\%) developed renal failure.

Conclusion: The results of the present study are better than those from most other series. The majority of children with IgAN in this study were admitted with recurrent macroscopic hematuria and found to have a good prognosis. We suggest that children with IgAN have a good prognosis in the first 5-year follow-up period.

Keywords: immunoglobulin A nephropathy, childhood, prognosis

\section{Introduction}

Immunoglobulin A (IgA) nephropathy (IgAN) is the most common form of glomerulonephritis in children and adolescents worldwide. ${ }^{1,2}$ The disease was described as a new clinical entity in $1968 .^{2}$ Kidney biopsy is essential for diagnoses that show a markedly expanded mesangium filled with IgA. The clinical presentation of IgAN varies across the spectrum of initial renal manifestations, ranging from microscopic hematuria to end-stage renal disease (ESRD). The prognosis was initially considered to be benign in children but long-term studies have shown that IgAN in children can follow the same progression as in adults. ${ }^{3,4}$ Furthermore, previous studies on this subject have shown that ESRD will occur in $6 \%-43 \%$ of patients with $\operatorname{IgA}$ nephropathy over a period of 10 years. Several factors affect the prognosis in patients with IgA nephropathy, such as elevated serum creatinine, heavy proteinuria, hypertension, and sex (with males more likely to have the disease). ${ }^{5}$

The aim of this study was to evaluate patients from a single center to describe the clinical features, treatments, and follow-up results of those with the disease.
Correspondence: Ipek Kaplan Bulut Ege University, Faculty of Medicine, Department of Pediatric Nephrology, 35100 , Izmir, Turkey

$\mathrm{Tel}+905336596809$

Fax+90232390I357

Email ikaplanbulut@gmail.com 


\section{Patients and methods}

This is a retrospective data study of all children with IgAN hospitalized at the Ege University, Faculty of Medicine, Department of Pediatric Nephrology, from January 1991 to December 2005. Patients who had a histopathologically proven diagnosis of IgAN and were followed up for at least 5 years were included in the study. All medical records were reviewed with emphasis on presenting symptoms, physical exam findings, laboratory data, therapies, and outcome. The local ethical committe approved all procedures in this study.

For each patient, the following information was completed from medical charts: patient age at time of biopsy, sex, serum creatinine $(\mathrm{mg} / \mathrm{dL})$, serum IgA levels; urinary protein excretion ( $\mathrm{g} / 24$ hours or protein/creatinine ratio); presence or absence of gross and/or microscopic hematuria; and presence or absence of hypertension (blood pressure $>95$ th percentile for age, gender, and height on repeated measurements). Hematuria was defined as a small amount (+) of hemoglobin on dipstick testing or greater than five red blood cells per high-power microscopic field in a centrifuged specimen.

Proteinuria was defined as a small amount of protein (+) on dipstick testing or proteinuria greater than $0.5 \mathrm{~g} / 1.73 \mathrm{~m}^{2} /$ day obtained from urine collected over 24 hours. The Schwartz formula was used to estimate creatinine clearance from the serum creatinine and height. Low creatinine clearance was defined as an estimated glomerular filtration rate (GFR) of $<60 \mathrm{~mL} / \mathrm{min}$ per $1.73 \mathrm{~m}^{2}$ body surface area. These patients were classified according to the initial clinical presentation. ${ }^{6}$ This classification was used as follows: microscopic hematuria \pm proteinuria; macroscopic hematuria; nephritic syndrome (hematuria, decrease in GFR, oliguria, hypertension, edema); nephrotic syndrome (proteinuria $>40 \mathrm{mg} / \mathrm{m}^{2} / \mathrm{h}$, hypoalbuminemia, hyperlipidemia, and edema). Histopathologic examination was done by the same pathologist. Renal biopsies, all of which contained at least ten glomeruli, were processed for light and immunofluorescence microscopy. In light microscopy, biopsies were graded from I to $\mathrm{V}$ in increasing severity according to the Hass classification ${ }^{7}$ grades as follows: Grade I, normal histology or mild increase in mesangial matrix, without segmental lesions; Grade II, focal and segmental glomerular sclerosis, without glomerular hypercellularity or crescents; Grade III, focal (involving $\leq 50 \%$ of glomeruli present, exclusive of globally sclerotic glomeruli) mesangial and/or endocapillary proliferative glomerulonephritis; Grade IV, diffuse (involving $>50 \%$ of glomeruli present, exclusive of globally sclerotic glomeruli) mesangial and/or endocapillary proliferative glomerulonephritis; Grade $\mathrm{V}$, advanced chronic glomerulonephritis, characterized by $\geq 40 \%$ globally sclerotic glomeruli and/or $\geq 40 \%$ interstitial fibrosis/tubular atrophy in the cortical tissue present, regardless of other histologic features. Mesengial IgA deposits are the defining hallmark of the disease in immunofluorescence microscopy.

Patients were placed into one of three treatment groups, as follows: (1) patients with recurrent macroscopic hematuria without proteinuria, and with normal kidney function, were treated with nonspecific therapy (omega-3:fish oil); (2) patients with proteinuria (range $0.5-3 \mathrm{~g} / 1.73 \mathrm{~m}^{2} /$ day) with or without microscopic hematuria had angiotensin converting enzyme inhibitors (ACEIs) and fish oil; and (3) patients with nephrotic syndrome had corticosteroid therapy (prednisolone or pulse methylprednisolone). Cytotoxic therapy was given when no response was observed following corticosteroid therapy.

Clinical outcome was graded as follows: A, normal (no hypertension, no urinary abnormality and no protein excretion and normal plasma creatinine concentration); $\mathrm{B}$, minor urinary abnormalities (proteinuria $<1 \mathrm{~g} / 1.73 \mathrm{~m}^{2} /$ day with or without microscopic-recurrent macroscopic hematuria); $\mathrm{C}$, active renal disease (proteinuria $>1 \mathrm{~g} / 1.73 \mathrm{~m}^{2} /$ day and/or elevated plasma creatinine level); or $\mathrm{D}$, renal insufficiency (GFR $<60 \mathrm{~mL} / \mathrm{min} / 1.73 \mathrm{~m}^{2}$ ).

\section{Statistical analysis}

Categorical data and proportions were compared using the chi-square test or Fisher's exact test, as indicated. Means were compared by Student's $t$-test, and medians were compared using the Mann-Whitney U test. The Kruskal-Wallis test was applied to the ordinal variables. A value of $P<0.05$ was considered statistically significant. SPSS (v 11.0; SPSS Inc, Chicago, IL) software was used for statistical analysis.

\section{Results}

A total of 39 patients were included in the study. The mean follow-up time ( \pm standard deviation [SD]) was $10.4 \pm 3.51$ (range 5-16) years. Twenty-nine (74.4\%) were male and ten (25.6\%) female. Males were affected more often than females (males:females $=3: 1)$. The mean age $( \pm \mathrm{SD})$ of the patients at onset of the disease was $9.5 \pm 3.75$ years (range 4-17). Elevated serum creatinine and hypertension were detected in $7.7 \%$ and $10.3 \%$ of patients, respectively. Nineteen (48.7\%) patients presented with recurrent macroscopic hematuria, ten $(25.6 \%)$ with microscopic hematuria \pm proteinuria, six (15.4\%) with nephritic syndrome, and four (10.3\%) with nephrotic syndrome. The demographic and clinical findings of patients are shown in Table 1. 
Table I The demographic and clinical features in 39 children with immunoglobulin A nephropathy

\begin{tabular}{ll}
\hline Features & $\mathbf{n}$ \\
\hline Age at initial onset (year) & $9.5 \pm 3.75(4-17)$ \\
Mean \pm SD (range) & \\
Sex & $29 / 10$ \\
Male/female & $16(41)$ \\
High levels of serum IgA (n [\%]) & $19(48.7)$ \\
Recurrent macroscopic hematuria (n [\%]) & $10(25.6)$ \\
Hematuria \pm proteinuria (n [\%]) & $6(15.4)$ \\
Nephritic syndrome $(\mathrm{n}[\%])$ & $4(10.3)$ \\
Nephrotic syndrome $(\mathrm{n}[\%])$ &
\end{tabular}

All patients underwent renal biopsy. Immunofluorescence study showed IgA deposition in the glomerular mesangium in all specimens. Light microscopic findings of biopsies were: $22(56.4 \%)$ patients, Grade I; four (10.2\%), Grade II; eleven (28.3\%), Grade III; and two (5.1\%), Grade IV. There were no patients in advanced chronic glomerulonephritis (Grade V).

All patients in the study received fish oil after diagnosis. Twenty-nine (74.4\%) patients were treated with ACEIs, and ten $(25.6 \%)$ with corticosteroids. Three $(7.7 \%)$ patients had corticosteroid plus cytotoxic agents including cyclosporine-A, cyclophosphamide, and azathiopurine. Four patients with nephrotic syndrome, three patients with nephritic syndrome, two patients with hematuria \pm proteinuria, and one patient with recurrent macroscopic hematuria were given steroid therapy (Table 2).

At the end of follow-up time, 18 (46.1\%) patients were normal, 15 (38.5\%) had minor urinary abnormalities, three (7.7\%) had active renal disease, and three (7.7\%) developed renal failure. The relationship between initial clinical findings, biopsy findings, and outcomes of the patients is shown in Table 3.

All patients were evaluated according to risk factors at admission. Three patients with elevated serum creatinine levels presented with recurrent macroscopic hematuria. These patients had Grade III renal biopsy specimens. Two of these patients entered remission while one had minor urinary abnormalities at last follow-up time.

Four $(10.3 \%)$ of 39 patients had hypertension on admission. Two patients presented with recurrent macroscopic hematuria, and two with microscopic hematuria \pm proteinuria. Three patients had Grade I, one had Grade IV. One of these four patients achieved remission, one had minor urinary abnormalities, one had active renal disease, and one developed renal failure at last follow-up (Table 4).

At onset, 20 patients had proteinuria ( $>1 \mathrm{~g}$ /day). Two of these had active renal disease while two developed renal failure at last follow-up time.

\section{Discussion}

This study evaluated initial clinical features, treatment modalities, and outcome in children with IgAN. Thirty-nine patients with IgAN were retrospectively reviewed over a 15-year period.

IgAN occurs at all ages but is most common during the second and third decades of life and uncommon in those under the age of 3 years. ${ }^{6,8}$ The peak incidence of IgA nephropathy in children occurs between 9 and 10 years of age. ${ }^{2}$ In the present study, the mean age at disease onset was $9.5 \pm 3.7$ years. Primary IgAN is more frequent in males than females. ${ }^{2}$ The male to female ratio in this study was $3: 1$.

The clinical presentation of IgAN is not pathognomonic. Asymptomatic microscopic hematuria can be found in $60 \%$ of subjects. Recurrent macroscopic hematuria continues to occur in $20 \%-80 \%$ of these patients. ${ }^{1,9,10}$ Some cases may present with acute nephritic syndrome and acute renal failure. ${ }^{6}$ In the present study, patients presented with recurrent macroscopic hematuria, microscopic hematuria \pm proteinuria, nephritic syndrome, and nephrotic syndrome $(48.7 \%, 25.6 \%$, $15.4 \%, 10.3 \%$, respectively). It is suggested that recurrent

Table 2 Outcome of patients given steroid treatment

\begin{tabular}{|c|c|c|c|}
\hline Initial presentation & & Treatment & Outcome* \\
\hline \multirow[t]{3}{*}{ Nephrotic syndrome $(n=4)$} & 2 patients & ACEls + fish oil + steroid + CA** & $\mathrm{D}$ \\
\hline & I patient & ACEls + fish oil + steroid & C \\
\hline & I patient & ACEls + fish oil + steroid & A \\
\hline \multirow[t]{2}{*}{ Nephritic syndrome $(n=3)$} & 2 patients & ACEls + fish oil + steroid & B \\
\hline & I patient & ACEls + fish oil + steroid & A \\
\hline \multirow[t]{2}{*}{ Hematuria \pm proteinuria $(n=2)$} & I patient & ACEls + fish oil + steroid + CA** & $\mathrm{D}$ \\
\hline & I patient & ACEls + fish oil + steroid & $\mathrm{C}$ \\
\hline Recurrent macroscopic hematuria $(\mathrm{n}=1)$ & I patient & ACEls + fish oil + steroid & C \\
\hline
\end{tabular}

Notes: *Outcome: A, normal; B, minor urinary abnormalities; C, active renal disease; D, end-stage renal disease; **cytotoxic agents (CA): cyclosporine-A, cyclophosphamide, azathiopurine. 
Table 3 Clinical findings, biopsy findings, and outcome results of all patients

\begin{tabular}{llllll}
\hline Initial clinical findings & Biopsy & \multicolumn{5}{l}{ Outcome* } \\
\cline { 2 - 6 } & & A & B & C & D \\
\hline Recurrent macroscopic & Grade I & 6 & 4 & - & - \\
hematuria $(\mathrm{n}=19)$ & Grade II & $\mathrm{I}$ & $\mathrm{I}$ & - & - \\
\multirow{3}{*}{ Hematuria \pm proteinuria } & Grade III & 2 & 5 & - & - \\
$(\mathrm{n}=10)$ & Grade I & 3 & 2 & $\mathrm{I}$ & $\mathrm{I}$ \\
& Grade III & 2 & - & - & - \\
Nephritic syndrome $(\mathrm{n}=6)$ & Grade IV & - & $\mathrm{I}$ & - & - \\
& Grade I & 3 & $\mathrm{I}$ & - & - \\
Nephrotic syndrome $(\mathrm{n}=4)$ & Grade II & - & $\mathrm{I}$ & $\mathrm{I}$ & - \\
& Grade I & $\mathrm{I}$ & - & - & - \\
& Grade III & - & - & $\mathrm{I}$ & I \\
& Grade IV & - & - & - & I \\
\hline
\end{tabular}

Note: *Outcome: A, normal; B, minor urinary abnormalities; C, active renal disease; $D$, end-stage renal disease.

macroscopic hematuria is the most important presentation and seen more often than acute renal failure, a result similar to studies from Europe and United States. ${ }^{6}$

Hypertension is infrequently present at disease onset and, when present, is usually mild to moderate in severity and relatively easy to control. ${ }^{1,2}$ Likewise, decreased renal function rarely presents at disease onset. ${ }^{9}$ Ronkainen et al reported that hypertension at onset was present in $13 \%$ of the patients. ${ }^{1}$ Hypertension at onset was not common in the present series: only four (10.3\%) of the 39 patients had hypertension. One of these developed ESRD and another one had persistent, active renal disease at the end of follow-up. Three $(7.7 \%)$ of the 39 patients had elevated serum creatinine at onset. Patients who present with hypertension at disease onset should receive careful follow-up.

In the literature, higher serum IgA levels were detected in $35 \%-70 \%$ of patients with IgAN. ${ }^{6,10}$ This marker may suggest the possible presence of disease. High levels of $\operatorname{IgA}$ are found during the phases of clinical activity. ${ }^{11}$ In the present study, $41 \%$ of patients had elevated serum IgA at onset of disease.
The range of pathologic features of IgAN has been well described. Hass found that the most common histological lesions are focal proliferative glomerulonephritis, accounting for between $40 \%$ and $50 \%$ in both adults and children. ${ }^{7}$ In the Hass study, biopsies showing normal histology were more common in children than in adults $(27 \%$, $14 \%$, respectively). ${ }^{7}$ In immunofluorescence microscopy, mesengial diffuse IgA deposits are the defining hallmark of the disease. ${ }^{12}$ Immunoglobulin $\mathrm{G}$, immunoglobulin $\mathrm{M}$, and Complement 3 deposition may accompany $\operatorname{IgA} .{ }^{13}$ In the present study, renal biopsy was performed in all patients. The immunofluorescence study showed IgA deposition in the glomerular mesangium in all specimens. Minimal histologic lesion (Grade I) was most commonly seen in the patients $(56.4 \%)$. Focal proliferative glomerulonephritis (Grade III) and focal segmental glomerular sclerosis without proliferation (Grade II) accounted for $28.4 \%$ and $10.2 \%$, respectively. Eleven patients in the study had diffuse proliferative findings (Grade III) and seven presented with recurrent macroscopic hematuria at onset of disease. These results suggest that first clinical findings are not related to renal biopsy findings. Therefore, patients with IgAN should undergo renal biopsy irrespective of initial symptoms.

The optimal approach to the treatment of IgA nephropathy is still uncertain. There are four separate approaches to $\operatorname{IgA}$ nephropathy: ${ }^{14-16}$ (1) patients with recurrent macroscopic hematuria with normal renal function, no proteinuria, and no microscopic hematuria between episodes are treated with nonspecific therapy (fish oil); (2) patients with persistent proteinuria $\left(0.5-1 \mathrm{~g} / 1.73 \mathrm{~m}^{2} /\right.$ day $) \pm$ microscopic hematuria might be treated with ACEIs; (3) patients with proteinuria (1-3 g/1.73 $\mathrm{m}^{2} /$ day) persisting, despite ACEI/angiotensin receptor-blocker therapy, are treated with corticosteroid; and (4) patients with proteinuria (>3.5 g/1.73 $\mathrm{m}^{2} /$ day) and/or rising serum creatinine, and/or renal biopsy with more severe histologic findings may benefit from immunosuppressive

Table 4 Patients with elevated serum creatinine and hypertension at onset

\begin{tabular}{llllll}
\hline & Initial presentation & & Biopsy & Therapy & Outcome* \\
\hline Elevated serum & Recurrent macroscopic hematuria & Patient I & Grade III & ACEls + fish oil & B \\
creatinine & & Patient 2 & Grade III & ACEls + fish oil & A \\
& & Patient 3 & Grade III & ACEls + fish oil & A \\
Hypertension & Recurrent macroscopic hematuria & Patient I & Grade I & ACEls + fish oil & A \\
& & Patient 2 & Grade I & ACEls + fish oil & B \\
& Microscopic hematuria \pm proteinuria & Patient I & Grade IV & ACEls + fish oil + steroid & C \\
& & Patient 2 & Grade I & ACEls + fish oil + steroid + CA** & D \\
\hline
\end{tabular}

Notes: *Outcome: A, normal; B, minor urinary abnormalities; C, active renal disease; D, end-stage renal disease; ***cytotoxic agents (CA): cyclosporine-A, cyclophosphamide, azathiopurine.

Abbreviation: ACEls, angiotensin converting enzyme inhibitors. 
therapy in addition to corticosteroids. Donadio et al provided evidence that those patients who received omega 3 fatty acids (fish oil) for 2 years had a good prognosis. ${ }^{17}$ Hogg et al found parallel results. ${ }^{18}$ The lack of any significant side-effects and the potential for benefit make omega 3 fatty acid preparations an attractive initial option for patients with IgAN and proteinuria. ${ }^{19}$ Previous studies have shown that ACEIs alone or with angiotensin receptor-blocker therapy prevent progression of renal disease in patients with IgAN. ${ }^{20,21}$

All patients in the present study were treated with fish oil after diagnosis. Twenty-nine of 39 patients (74.4\%) were treated with ACEIs, and ten (25.6\%) with corticosteroids. Three $(7.7 \%)$ patients had corticosteroid plus cytotoxic agents including cyclosporine-A, cyclophosphamide, and azathiopurine. Four patients with nephrotic syndrome, three patients with nephritic syndrome, two patients with hematuria \pm proteinuria and one patient with recurrent macroscopic hematuria were given steroid therapy.

IgAN was initially thought to follow a benign course, but it is now recognized that slow progression to ESRD occurs in up to $50 \%$ of affected patients. ${ }^{16,22}$ The remaining patients enter a sustained clinical remission or have persistent low-grade hematuria or proteinuria. ${ }^{22}$ However, the prognosis is quite variable and the outcome difficult to predict with accuracy in individual patients. In the European Renal Association chronic dialysis program for young adults, $22 \%$ of patients were under 30 years of age. ${ }^{23}$ Short-term follow-up studies have shown a better prognosis for children, although long-term follow-up shows that $\operatorname{IgAN}$ in children is as progressive as in adults..$^{4,24,25}$ Long-term studies have concluded that complete remission occurs in 5\%-30\% of patients. ${ }^{10}$ The majority of children with IgAN in this study admitted with macroscopic hematuria were found to have a good prognosis. In this study, 18 patients (46.1\%) entered remission, which is a significantly higher proportion than in other studies reported to date. ${ }^{14,15}$

In 2004, the International IgA Nephropathy Network and the Renal Pathology Socienty established a working group to develop an international consensus on practical histopathological classification of IgAN ${ }^{26,27}$ The Oxford classification is the largest-scale histopathological study of IgA nephropathy. ${ }^{26,27}$ This classification provides substantially stronger evidence for a histopathological grading system to predict renal prognosis of IgA nephropathy. Compared with the previous classifications, in the present study the Oxford classification was found to be practical and useful.

In children with progressive IgAN, the clinical course is often slow. ${ }^{16}$ Among the factors that determine progression over the years are reduced renal function, persistent hypertension, and proteinuria at onset. ${ }^{28-30}$ Four patients had hypertension at onset. One of these achieved remission, one had minor urinary abnormalities, and one had active renal disease while one developed renal failure. Twenty patients had proteinuria $(>1 \mathrm{~g} /$ day) at onset. Two of these had active renal disease while two developed renal failure. Patients initially presenting with high serum creatinine levels, excepting those who had nephritic presentations, did not show poor prognosis, as the creatinine levels of all these patients were regressed to normal ranges shortly after disease onset. None had active renal disease, or developed renal failure at last follow-up. However, the authors believe that careful monitoring of these patients should continue.

\section{Conclusion}

The outcome results of the patients in this study are better than those reported in most other studies. Early diagnosis and treatment of IgAN in childhood may bring important benefits and a favorable prognosis. The majority of children with $\mathrm{IgAN}$ in this study were admitted with recurrent macroscopic hematuria, which was found to have a good prognosis. In addition, this study has shown that histopathological findings do not correlate with the course of the disease and progress of chronic renal failure. Steroids are still important agents in the treatment of IgA nephropathy. If patients with hypertension, elevated creatinine levels, or proteinuria are identified and receive early treatment, the course of disease can be modified and most patients will have a favorable prognosis.

\section{Disclosure}

The authors declare no conflicts of interest in this work.

\section{References}

1. Ronkainen J, Ala-Houhala M, Autio-Harmainen H, et al. Long term outcome 19 years after childhood IgA nephritis: a retrospective cohort study. Pediatr Nephrol. 2006;21(9):1266-1273.

2. Chan JC, Trachtman H. Modulating the progression in IgA nephropathy. Nephron Clin Pract. 2006;104(1):c61-c68.

3. Wyatt RJ, Julian BA, Bhathena DB, Mitchell BL, Holland NH, Malluche HH. IgA nephropathy: presentation, clinical course, and prognosis in children and adults. Am J Kidney Dis. 1984;4(2):192-200.

4. Wyatt RJ, Krichevsky SB, Woodford SY, et al. IgA nephropathy: longterm prognosis for pediatric patients. J Pediatr. 1995;127(6):913-919.

5. Wakai K, Kawamura T, Endoh M, et al. A scoring system to predict renal outcome in IgA nephropathy: from a nationwide prospective study. Nephrol Dial Transplant. 2006;21(10):2800-2808.

6. Yoshikawa N. Immunglobulin A nephropathy. In: Avner ED, Harmon WE, Niaudet P, editors. Pediatric Nephrology. Philadelphia, PA: Lippincott Williams and Wilkins; 2004:615-628.

7. Haas M. Histology and immunohistology of IgA nephropathy. J Nephrol. 2005;18(6):676-680

8. Kaplan BS. IgA nephropathy. In: Kaplan BS, Meyers KE, editors. Pediatric Nephrology and Urology: The Requisites in Pediatrics. Maryland Heights, MO: Mosby; 2004:137-140. 
9. Fofi C, Pecci G, Galliani M, et al. IgA nephropathy: multivariate statistical analysis aimed at predicting outcome. J Nephrol. 2001;14(4): 280-285.

10. Coppo R, D’Amico G. Factors predicting progression of IgA nephropathies. J Nephrol. 2005;18(5):503-512.

11. Coppo R, Amore A, Gianoglio B, et al. Macromolecular IgA and abnormal $\operatorname{IgA}$ reactivity in sera from children with IgA nephropathy. Italian Collaborative Paediatric IgA Nephropathy Study. Clin Nephrol. 1995;43(1):1-13.

12. Allen AC, Barratt J, Feehally J. Immunoglobulin A nephropathy. In: Neilson EG, Couser WG, editors. Immunologic Renal Disease. Philadelphia, PA: Lippincott Williams and Wilkins; 2001:931-947.

13. Barratt J, Feehally J. IgA nephropathy. J Am Soc Nephrol. 2005;16(7): 2088-2097.

14. Barratt J, Feehally J. Treatment of IgA nephropathy. Kidney Int. 2006;69(11):1934-1938.

15. Appel GB, Waldman M. The IgA nephropathy treatment dilemma. Kidney Int. 2006;69(11):1939-1944.

16. Coppo R, Amore A. IgA nephropathy. In: Geary DF, Schaefer F, editors. Comprehensive Pediatric Nephrology. Maryland Heights, MO: Mosby; 2008:291-299.

17. Donadio JV Jr, Bergstralh EJ, Offord KP, Spencer DC, Holley KE. A controlled trial of fish oil in IgA nephropathy. Mayo Nephrology Collaborative Group. N Engl J Med. 1994;331(18):1194-1199.

18. Hogg R, Fitzgibbons L, Atkins C, Bay R. Efficacy of omega-3 fatty acids in children and adults with IgA nephropathy is dosage- and size dependent: report from Southwest Pediatric Nephrology Study Group. Clin J Am Soc Nephrol. 2006;1(6):1167-1172.

19. Hogg R. IgA nephropathy: what's new? Pediatr Nephrol. 2007;22(11): 1809-1814.

20. Praga M, Gutierrez E, Gonzalez E, Morales E, Hernandez E. Treatment of IgA nephropathy with ACE inhibitors: a randomized and controlled trial. J Am Soc Nephrol. 2003;14:1578-1583.
21. Coppo R, Peruzzi L, Amore A, et al. IgACE: a placebo-controlled randomized trial of ACE-inhibitors (ACE-I) in children and young people with IgA nephropathy and moderate proteinuria. J Am Soc Nephrol. 2007;18(6): 1880-1888.

22. Geddes CC, Rauta V, Gronhagen-Riska C, et al. A tricontinental view of IgA nephropathy. Nephrol Dial Transplant. 2003;18(8):1541-1548.

23. Fassbinder W, Brunner FP, Brynger H, et al. Combined report on regular dialysis and transplantation in Europe, XX, 1989. Nephrol Dial Transplant. 1991;6 Suppl 1:5-35.

24. Schena FP. A retrospective analysis of the natural history of primary IgA nephropathy worldwide. Am J Med. 1990;89(2):209-215.

25. Linne T, Berg U, Bohman SO, Sigstrom L. Course and long term outcome of idiopathic IgA nephropathy in children. Pediatr Nephrol. 1991;5(4):383-386.

26. Working Group of the International IgA Nephropathy Network and the Renal Pathology Society; Cattran DC, Coppo R, Cook HT, et al. The Oxford classification of IgA nephropathy: rationale, clinicopathological correlations, and classification. Kidney Int. 2009;76(5):534-545.

27. Working Group of the International IgA Nephropathy Network and the Renal Pathology Society; Roberts IS, Cook HT, Troyanov S, et al. The Oxford classification of IgA nephropathy: pathology definitions, correlations, and reproducibility. Kidney Int. 2009;76(5):546-556.

28. Hogg RJ, Silva FG, Wyatt RJ, Reisch JS, Argyle JC, Savino DA. Prognostic indicators in children with IgA nephropathy-report of the Southwest Pediatric Nephrology Study Group. Pediatr Nephrol. 1994;8(1):15-20.

29. Ibels LS, Gyory AZ. IgA Nephropathy: analysis of the natural history, important factors in the progression of renal disease, and a review of the literature. Medicine (Baltimore). 1994;73(2):79-102.

30. Bartosik LP, Lajoie G, Sugar L, Cattran DC. Predicting progression in IgA nephropathy. Am J Kidney Dis. 2001;38(4):728-735.

\section{Publish your work in this journal}

The International Journal of Nephrology and Renovascular Disease is an international, peer-reviewed open-access journal focusing on the pathophysiology of the kidney and vascular supply. Epidemiology, screening, diagnosis, and treatment interventions are covered as well as basic science, biochemical and immunological studies. The journal welcomes original research, clinical studies, reviews \& evaluations, expert opinion and commentary, case reports and extended reports. The manuscript management system is completely online and includes a very quick and fair peerreview system, which is all easy to use. Visit http://www.dovepress.com/ testimonials.php to read real quotes from published authors. 